\title{
Adverse drug reactions and interactions: first reports and special situations
}

C) Springer Nature Switzerland AG 2020

Table 1 contains an overview of first published case reports of adverse drug reactions and interactions, as well as selected case reports of adverse drug reactions in special situations, as recently identified by Adis Insight. A database for drug research, disease treatment and decision making,
Adis Insight monitors a variety of global sources to identify new drug-related data, which are then reviewed, assessed, validated, categorized and summarized by scientists in the editorial team.

Table 1 Adverse drug reactions and interactions: first and selected special-situation case reports recently identified by Reactions Weekly

\begin{tabular}{|c|c|c|c|}
\hline Drug & ADR or outcome & $\begin{array}{l}\text { Pt gender (age): condition } \\
\text { treated }\end{array}$ & References \\
\hline \multicolumn{4}{|l|}{ First reports of ADRs } \\
\hline Ceftazidine & Urinary calculi (serious) & $M(8 y): N R$ & $\begin{array}{l}\text { Gao P, Liu Z, Yang H, et al. Ceftazidime-related urinary } \\
\text { calculi in a young boy: a case report. J Int Med Res. } \\
\text { 2020;48(4) }\end{array}$ \\
\hline Cimicifuga & $\begin{array}{l}\text { Superior sagittal and right } \\
\text { transverse sinus thrombo- } \\
\text { sis (serious) }\end{array}$ & $\begin{array}{l}\text { F (49 y): menopausal } \\
\text { symptoms }\end{array}$ & $\begin{array}{l}\text { Mittal A, Iliceto A, Yegneswaran B. Herbopathy-induced } \\
\text { cephalalgia: remedy gone wrong. J Coll Physicians Surg } \\
\text { Pak. 2019;18(3):148-9 }\end{array}$ \\
\hline Cinacalcet & $\begin{array}{l}\text { Cystic parathyroidal ade- } \\
\text { noma with rapid growth }\end{array}$ & $\begin{array}{l}\text { F (56 y): primary hyper- } \\
\text { parathyroidism }\end{array}$ & $\begin{array}{l}\text { Werner C, Lupp A, Mtuka-Pardon G, et al. Case of } \\
\text { report of a cystic parathyroidal adenoma with rapid } \\
\text { growth induced by cinacalcet. BMC Endocr Disord. } \\
\text { 2020;20(1):53 }\end{array}$ \\
\hline Cissus quadrangularis & $\begin{array}{l}\text { Thrombocytopenia (seri- } \\
\text { ous) }\end{array}$ & M (65 y): backache & $\begin{array}{l}\text { Kulkami MJ, Bhat AS. Ciccus quadrangularis-induced } \\
\text { thrombocytopenia in a renal allograft recipient. Exp Clin } \\
\text { Transplant. 2020;18(2):250-1 }\end{array}$ \\
\hline Levetiracetam & $\begin{array}{l}\text { Minimal change disease } \\
\quad \text { (serious) }\end{array}$ & $\begin{array}{l}\text { M (31 y): generalized } \\
\text { tonic-clonic seizures }\end{array}$ & $\begin{array}{l}\text { Tarun TN, Tarun T, Malhotra K. First case report of leveti- } \\
\text { racetam induced MCD: unicorn in the barn [abstract no. } \\
\text { 262]. Am J Kidney Dis. 2020;75(4):612 }\end{array}$ \\
\hline Phytomenadione & Anetoderma & $\begin{array}{l}\text { F (1 y): neurofibromatosis } \\
\text { type } 1\end{array}$ & $\begin{array}{l}\text { Esposito I, Guerriero C, Leoni C, et al. One case of } \\
\text { anetoderma post-vitamin K1 injection in a newborn. Int } \\
\text { J Dermatol. 2020;59(5):e168-e169 }\end{array}$ \\
\hline Tamsulosin & Fixed-drug eruption & $\begin{array}{l}\text { M (54 y); prostatic hyper- } \\
\text { plasia }\end{array}$ & $\begin{array}{l}\text { Montazer F, Jahani Amiri K, Ahmadi A, et al. A first } \\
\text { case of fixed drug eruption due to tamsulosin. J Cosmet } \\
\text { Dermatol. 2020;19(5):1143-5 }\end{array}$ \\
\hline \multicolumn{4}{|l|}{ In utero drug exposure } \\
\hline $\begin{array}{l}\text { Elvitegravir-containing } \\
\text { ARV therapy }\end{array}$ & $\begin{array}{l}\text { Fetal death in } 2 \text { of } 134 \\
\text { pregnancies (serious) }\end{array}$ & $2 \mathrm{~F}$ (NR): HIV & $\begin{array}{l}\text { Badell ML, Sheth AM, Momplaisir F, et al. A multicentre } \\
\text { analysis of elvitegravir use during pregnancy on HIV } \\
\text { viral suppression and perinatal outcomes. Open Forum } \\
\text { Infect Dis. } 2019 ; 6(4)\end{array}$ \\
\hline
\end{tabular}


Table 1 (continued)

\begin{tabular}{|c|c|c|c|}
\hline Drug & ADR or outcome & $\begin{array}{l}\text { Pt gender (age): condition } \\
\text { treated }\end{array}$ & References \\
\hline $\begin{array}{l}\text { Paracetamol (high } \\
\text { dose) + sertraline }\end{array}$ & $\begin{array}{l}\text { Persistent pulmonary hyper- } \\
\text { tension and premature } \\
\text { PDA closure in } 1 \text { twin at } \\
\text { 33-wk GA (serious) }\end{array}$ & $\begin{array}{l}\text { F (NR): panic attacks and } \\
\text { severe headache }\end{array}$ & $\begin{array}{l}\text { Corti CG, Faiola S, Lanna MM, et al. Monochorionic } \\
\text { diamniotic twin pregnancy complicated by discordant } \\
\text { premature closure of ductus arteriosus. Clin Case Rep. } \\
2020 ; 8(4): 685-9\end{array}$ \\
\hline Valproic acid & $\begin{array}{l}\text { Spina bifida }+ \text { other } \\
\text { congenital anomalies } \\
\text { (pregnancy due to COC } \\
\text { failure; serious) }\end{array}$ & $\begin{array}{l}\text { F (30): schizoaffective } \\
\text { disorder }\end{array}$ & $\begin{array}{l}\text { Suri R, Socol DK, Gitlin M. Standard of care: reasonable } \\
\text { but not perfect. Am J Psychiatry. 2020;177(3):210-3 }\end{array}$ \\
\hline \multicolumn{4}{|c|}{ ADR associated with a genetic factor } \\
\hline Progesterone implant & $\begin{array}{l}\text { Acute intermittent por- } \\
\text { phyria (serious) }\end{array}$ & F (23 y): contraception & $\begin{array}{l}\text { Tynan C, O'Dwyer G, Ahern T. Unexplained severe } \\
\text { abdominal pain, hyponatemia and visual scotoma: a case } \\
\text { report [abstract]. Ir J Med Sci. 2019;188a(Suppl 7):S71 }\end{array}$ \\
\hline \multicolumn{4}{|c|}{ COVID-19 in pts receiving immunosuppressive treatment } \\
\hline Fingolimod & COVID-19 (serious) & $\begin{array}{l}\text { F (47 y): relapsing-remit- } \\
\text { ting multiple sclerosis }\end{array}$ & $\begin{array}{l}\text { Foerch C, Friedauer L, Bauer B, et al. Severe COVD-19 } \\
\text { infections in a patient with multiple sclerosis treated } \\
\text { with fingolimod. Mult Scler Relat Dis. 2020;14:202180 }\end{array}$ \\
\hline \multicolumn{4}{|c|}{ Medication error, abuse, overdose or under-dose } \\
\hline Alprazolam & $\begin{array}{l}\text { Altered mental status }+ \\
\text { variable fetal heart rate } \\
\text { decelerations }\end{array}$ & $\begin{array}{l}\mathrm{F}(32 \mathrm{y})+\text { fetus }(\mathrm{GA} \\
41 \text { wk): overdose and } \\
\text { misuse during and post- } \\
\text { labour }\end{array}$ & $\begin{array}{l}\text { Berman DJ. A case of local anesthetic toxicity that wasn't: } \\
\text { lipid rescue from self-administered benzodiazepine over- } \\
\text { dose in labor. In J Obstet Anesth. 2020;42:109-11 }\end{array}$ \\
\hline Apixaban & $\begin{array}{l}\text { Femoral vein thrombus } \\
\text { (serious) }\end{array}$ & $\begin{array}{l}\text { M (43 y): unintentional } \\
\text { under-dose in an obese } \\
\text { pt (BMI 37.6) }\end{array}$ & $\begin{array}{l}\text { O'Riordan S, Wan Mahmood WA. Sub-therapeutic } \\
\text { dosing of direct oral anticoagulants in obese patients } \\
\text { with deep venous thrombosis [abstract]. Ir J Med Sci. } \\
\text { 2019;188(Suppl 7):S38-S39 }\end{array}$ \\
\hline Calcium carbamide & $\begin{array}{l}\text { RPLS + other toxicities } \\
\quad \text { (serious) }\end{array}$ & F (48): suicide attempt & $\begin{array}{l}\text { Penasco Y, Gonzalex-Castro A, Rodriguez-Borregan JC, } \\
\text { et al. Posterior reversible leukoencephalopathy after } \\
\text { cyanamide overdose. Neurologia. 2020;35(1):67-8 }\end{array}$ \\
\hline Citalopram & $\begin{array}{l}\text { No cardiac event during } \\
\text { 24-h post-overdose car- } \\
\text { diac telemetry }\end{array}$ & NR: intentional overdose & $\begin{array}{l}\text { Lee VR, Huang MT, Brambl WJ, et al. Asympto- } \\
\text { matic escitalopram and citalopram overdoses: 6-hour } \\
\text { observation period? [abstract no. 041]. J Med Toxicol. } \\
\text { 2020;16(2):127-8 }\end{array}$ \\
\hline Colchicine & $\begin{array}{l}\text { Multisystem organ failure }+ \\
\text { other toxicities (serious) }\end{array}$ & $\begin{array}{l}\mathrm{F}(33 \mathrm{y}) \text { : intentional } \\
\text { overdose }\end{array}$ & $\begin{array}{l}\text { Mulkareddy V, Sokach C, Bucklew E, et al. Colchi- } \\
\text { cine toxicity: the fatal masquerade. JACC Case Rep. } \\
\text { 2020;2(4):678-80 }\end{array}$ \\
\hline Etizolam & $\begin{array}{l}\text { Somnolence, ataxia }+ \text { other } \\
\text { benzodiazepine-related } \\
\text { symptoms }\end{array}$ & $\begin{array}{l}3 \text { children }(6-10 \text { y): con- } \\
\text { sumption of unlabelled } \\
\text { tablets appearing similar } \\
\text { to "Pez" candy }\end{array}$ & $\begin{array}{l}\text { Love JS, Thompson JA, Girardi F, et al. Candy coma: a } \\
\text { case series of etizolam exposures [abstract no. 044]. J } \\
\text { Med Toxicol. 2020;16(2):128 }\end{array}$ \\
\hline Hydroxychloroquine & $\begin{array}{l}\text { Tachycardia, hypotension }+ \\
\text { other toxicities (serious) }\end{array}$ & $\begin{array}{l}\mathrm{F}(15 \mathrm{y}) \text { : intentional } \\
\text { overdose }\end{array}$ & $\begin{array}{l}\text { Srihari P, Gao HT, Kreshak AA. Massive nonfatal pediat- } \\
\text { ric ingestion of hydroxychloroquine [abstract no. 023] . J } \\
\text { Med Toxicol. 2020;16(2):122-3 }\end{array}$ \\
\hline Ketamine & $\begin{array}{l}\text { Bradycardia, diaphoresis, } \\
\text { hypotension + other } \\
\text { toxicities (serious) }\end{array}$ & $\begin{array}{l}\text { M ( } 46 \text { y): error in } \\
\text { administration (IV push } \\
\text { instead of } 0.5-1 \mathrm{~h} \mathrm{IV} \\
\text { infusion) }\end{array}$ & $\begin{array}{l}\text { Emerling AD, Fisher J, Walrath B, et al. Rapid ketamine } \\
\text { infusion at an analgesic dose resulting in transient hypo- } \\
\text { tension and bradycardia in the emergency department. J } \\
\text { Spec Oper Med. 2020;20(1):31-3 }\end{array}$ \\
\hline
\end{tabular}

An ADR is serious (US FDA MedWatch definition) when the pt outcome is death, life threatening, hospitalization, disability, congenital anomaly or requires intervention to prevent permanent impairment or damage.

$A D R$ adverse drug reaction, $A R V$ antiretroviral, BMI body mass index, COC combined oral contraceptive, COVID-19 coronavirus disease 2019, $F$ female, $G A$ gestational age, $I V$ intravenous, $M$ male, mo months, $N R$ not reported, $p t$ patient, $R P L S$ reversible posterior leukoencephalopathy syndrome, $P D A$ patent ductus arteriosus, $w k$ weeks, $y$ years 